\title{
Morphological variations in pulmonary atresia with intact ventricular septum
}

\author{
JAMES R. ZUBERBUHLER ${ }^{1}$ AND ROBERT H. ANDERSON ${ }^{2}$ \\ From the Department of Paediatrics, Cardiothoracic Institute, Brompton Hospital, L.ondon; and Department \\ of Child Health, Institute of Child Health, Alder Hey Hospital, Liverpool
}

SUMMARY The morphological features of a series of 37 specimens of pulmonary atresia with intact ventricular septum were reviewed with particular emphasis on features which might influence the results of pulmonary valvotomy. The degree of right heart hypoplasia was quantified by measuring right and left heart dimensions and comparing them with 20 normal infant hearts. Right ventricular cavity size was usually smaller than normal but constituted a spectrum ranging from tiny to a dilated ventricle larger than normal. There was a positive correlation between triscuspid annular size and right ventricular size but no correlation between the size of the pulmonary artery and the right ventricle. Successful pulmonary valvotomy with subsequent adequate right ventricular function would have been precluded by a tiny right ventricular cavity or infundibular atresia in 14 specimens and by severe tricuspid stenosis or regurgitation in an additional 4. Severe right or left ventricular endocardial fibroelastosis may have adversely affected ventricular function in several others. Ten specimens displayed convex bulging of the left ventricular septal surface. If these anatomical findings are representative for the condition as a whole, they provide a good explanation for its disastrous prognosis.

The life of an infant born with pulmonary atresia and an intact ventricular septum is likely to be brief and stormy. Medical treatment is unavailing and survival depends upon the surgical establishment of a reliable source of pulmonary blood flow, either by a systemic-pulmonary artery shunt, by pulmonary valvotomy, or by both. Whatever techniques are employed, surgical mortality remains disappointingly high (Trusler et al., 1976). It is possible that the right heart hypoplasia known to be common with pulmonary atresia could account for these disappointing results. However, there is no agreement concerning the nature and frequency of this hypoplasia. Greenwold et al. (1956) believed that right ventricular cavity size could be classified as small or large and this view was endorsed by Davignon et al. (1961) and Celermajer et al. (1968). In contrast, others have contended that cavity size constitutes a spectrum (Gersony et al., 1967;

\footnotetext{
${ }^{1}$ Dr Zuberbuhler was a Visiting Fellow on leave of absence from the Children's Hospital, University of Pittsburgh, Pittsburgh, Pennsylvania, USA.

${ }^{2} \mathrm{Dr}$ Anderson is supported by the Joseph Levy Foundation and the British Heart Foundation.
}

Received for publication 13 February 1978
Bowman et al., 1971; Murphy et al., 1971). As cardiac morphology must influence surgical results, particularly if an effort is made to establish a communication between right ventricle and pulmonary artery, we have undertaken a review of the morphological features of pulmonary atresia, including measurements of right and left heart dimensions and their comparison with those of a series of normal infant hearts.

\section{Subjects and methods}

Thirty-seven infant hearts with pulmonary atresia and intact ventricular septum together with 3 hearts with critical pulmonary stenosis were found in the cardiopathological collections of the Brompton Hospital, Cardiothoracic Institute (London); Killingbeck Hospital (Leeds); Institute of Child Health, Alder Hey Hospital (Liverpool); and Grimsby General Hospital (South Humberside). These specimens were examined and compared with 20 normal infants' hearts from the Wilhelmina Gasthuis (Amsterdam). Emphasis was on those particular features which might have a bearing on selection of the optimal surgical approach and on its outcome; specifically, the size and morphological 


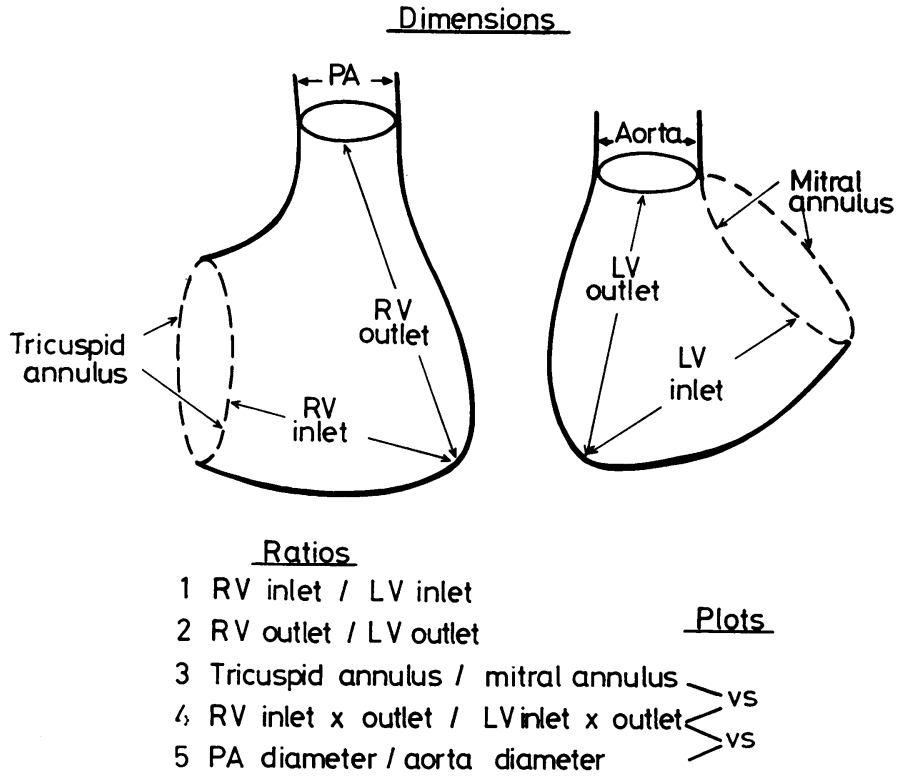

Fig. 1 Diagram showing measurements, ratios, and plots obtained. $R V$, right ventricle; $L V$, left ventricle; $P A$, pulmonary artery; vs, versus.

features of the tricuspid and pulmonary valves, the right ventricular cavity, and the pulmonary artery. The inlet and outlet dimensions of the right and left ventricles, the circumference of the tricuspid and mitral annuli, and the diameters of the pulmonary artery and aorta were measured (Fig. 1). Ratios between each right-sided measurement and its corresponding left-sided dimension were calculated as was the ratio of the product of the inlet and outlet dimensions of the right and left ventricles. The ratio of the products of right to left ventricular inlet and outlet measurements was plotted against both the tricuspid to mitral annulus ratio and the pulmonary to aortic diameter ratio.

Abnormalities of the tricuspid valve which might have influenced function, such as Ebstein's anomaly, leaflet dysplasia, or abnormal chordal or papillary muscle attachments were searched for and the likelihood of either regurgitation or of stenosis out of proportion to the annular or right ventricular size was noted.

In each specimen the right ventricular cavity size was subjectively graded as tiny, small, normal, or dilated. The cavity was judged to be tiny if deemed too small to sustain life were right ventricular to pulmonary artery continuity to be established. The categories small, normal, and dilated are self-explanatory. The presence of infundibular stenosis or atresia was noted. The presence of endocardial fibroelastosis, grossly visible myocardial sinusoids, and the degree of trabeculation of the

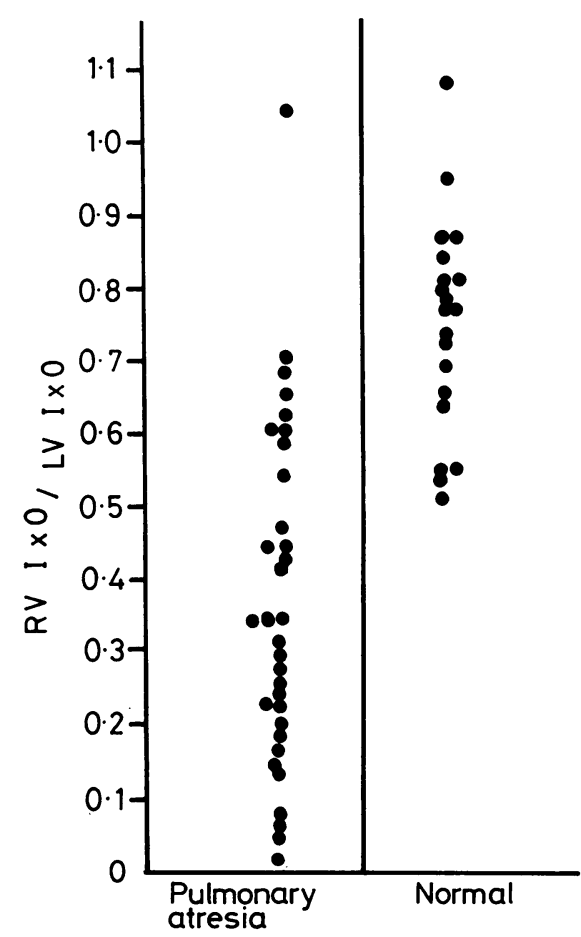

Fig. 2 Comparison of relative right ventricular cavity size in normal hearts with pulmonary atresia and intact ventricular septum. $R V I \times 0$, product of right ventricular inlet and outlet dimensions; $L V I \times 0$, product of left ventricular inlet and outlet dimensions. Measurements were possible in only 33 specimens. 
right ventricle were recorded. The myocardial thickness in the inlet, outlet, and apical portions of each ventricle was measured.

The morphological characteristics of the pulmonary valve itself were noted, including the number of cusps, extension of commissural ridges to the centre of the valve, and, in the case of critical pulmonary stenosis, the size of the pulmonary valve orifice. The length, outer diameter, and patency of the ductus arteriosus were noted, as was the presence or absence of left ventricular septal bulging.

\section{Results}

There was considerable variability in right ventricular cavity size in the group of normal hearts and an even greater range in specimens with pulmonary atresia. In general, the right ventricular cavity was smaller than normal with pulmonary atresia, but there was overlap between pulmonary atresia and normal as indicated by the ratios of the product of the inlet and outlet dimensions in right and left ventricles (Fig. 2). In all 12 cases judged to have a tiny right ventricle the infundibulum was either atretic or stenosed, atresia being present in 8 of the hearts. Infundibular atresia was also observed in 1 heart with a small right ventricle but in none of the hearts judged to have normal or large right ventricular cavity size. Infundibular stenosis, however, was found in 8 of 13 cases judged to have small right ventricles together with 1 heart with a normal-sized right ventricle and 1 heart with a large right ventricle. The right ventricle was of normal size in 2 specimens with critical pulmonary stenosis and large in the third. Grossly evident right ventricular endocardial fibroelastosis was found in 10 cases. There was little correlation with heart size, fibroelastosis being found in small and normal sized right ventricles as well as in tiny ones.

Right ventricular trabeculations were shallow and inconspicuous in most specimens, and myocardial sinusoids were grossly visible in several. In 20 hearts it was not possible accurately to measure myocardial thickness of both right and left ventricles in their apical and outlet portions, but this was possible in most specimens in the inlet portion. In two-thirds of the specimens with pulmonary atresia the right ventricular inlet wall was as thick or thicker than the left ventricular inlet wall.

In 11 specimens the pulmonary valve had been destroyed or greatly altered by surgery, and in 3 cases with a tiny main pulmonary artery no pulmonary valve leaflets were visible. In the remaining specimens 2 types of atretic pulmonary valve were seen. In the first type, prominent commissural ridges converged and met at the centre of the 'valve' (Fig. 3a). This type of pulmonary 'valve' was invariably associated either with infundibular atresia or with a severely stenotic or regurgitant tricuspid valve. The second type of pulmonary valve had commissural ridges present only at the periphery of the membranous pulmonary valve, the centre being smooth and bulging into the pulmonary trunk (Fig. 3b). In 1 specimen the pulmonary valve was bicuspid and in 1 quadricuspid. In each of the 3 cases with critical pulmonary stenosis, the pulmonary valve was thin and dome-

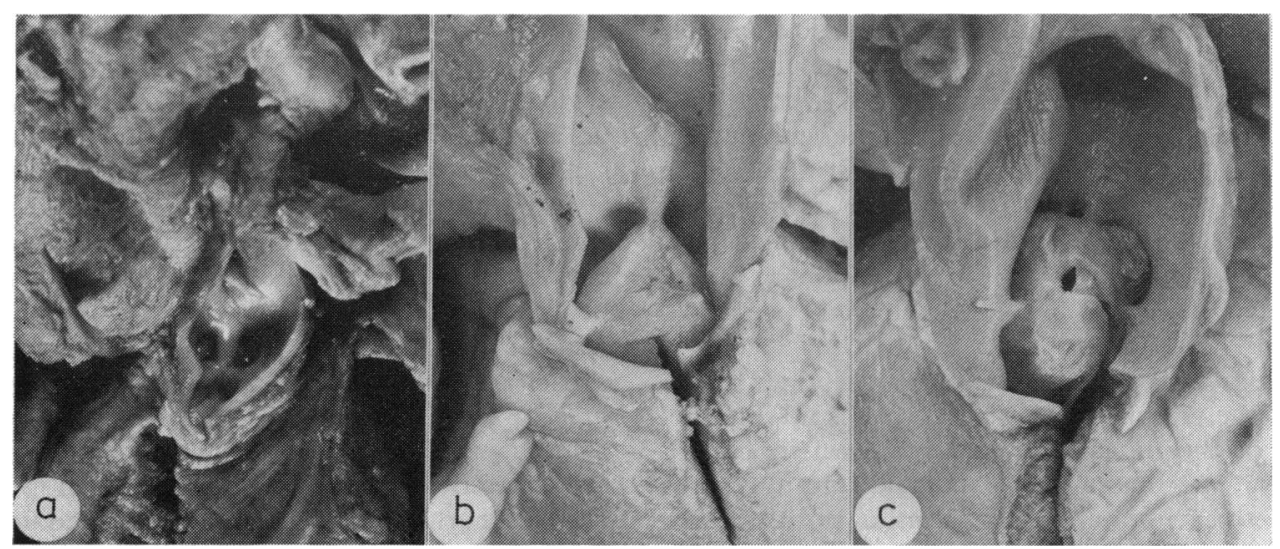

Fig. 3 The pulmonary 'valve'. (a) Commissural ridges extending to the centre of the 'valve'.

(b) Commissural ridges at the periphery of the valve. The centre is smooth and convex towards the pulmonary trunk. (c) Critical pulmonary stenosis with a tiny central orifice. Measurements were possible in only 36 specimens. 


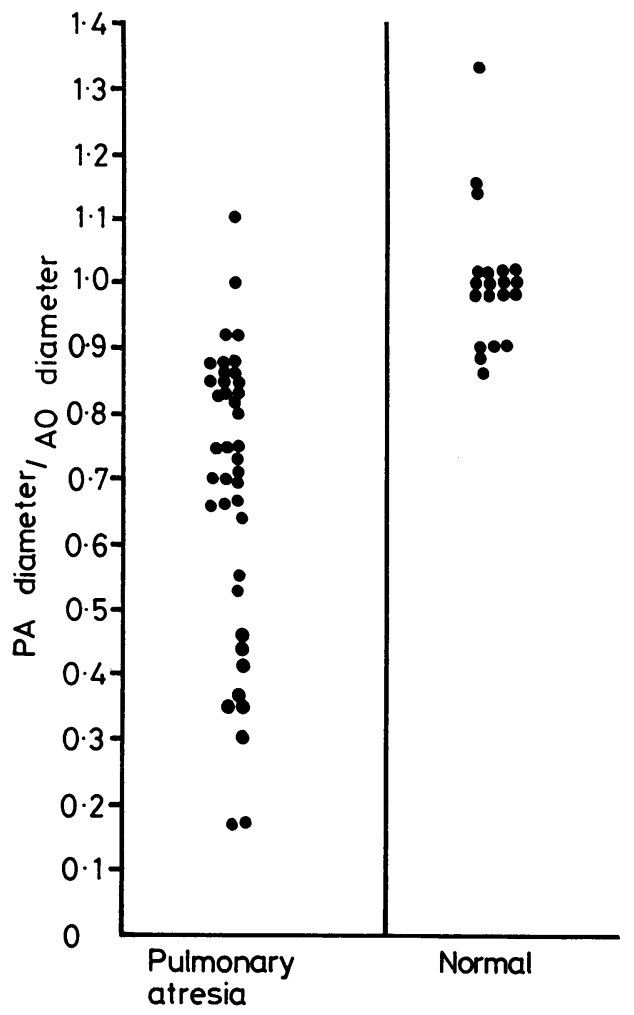

Fig. 4 Comparison of relative pulmonary artery size in normal hearts and in specimens with pulmonary atresia and pulmonary stenosis and with intact ventricular septum. AO, aorta; $P A$, pulmonary artery.

shaped with a central orifice less than $1 \mathrm{~mm}$ in diameter (Fig. 3c).

The size of the pulmonary artery, as judged from the ratio of pulmonary artery to aortic diameter, is shown in Fig. 4. Though the pulmonary artery size was small in a few specimens, the overlap with normal was large. The size of the pulmonary artery did not correlate with right ventricular cavity size, a tiny right ventricular cavity often supporting a normal-sized pulmonary artery (Fig. 5). In only 3 cases was the main pulmonary artery strikingly small and in each the right ventricle was tiny.

The size of the tricuspid annulus, as judged by the ratio of the circumference of the tricuspid annulus to that of the mitral annulus, is shown in Fig. 6. In most specimens the tricuspid annulus was smaller than normal. There was a positive correlation between the size of the tricuspid annulus and that of the right ventricular cavity $(R=$ $0 \cdot 84$ ). Tricuspid valve abnormalities found in this series are shown in the Table. The tricuspid

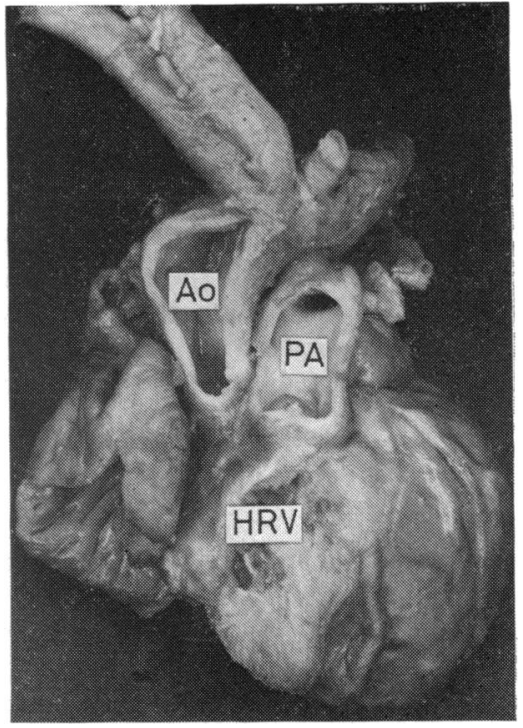

Fig. 5 A normal-sized pulmonary artery $(P A)$ arising from a very small right ventricle $(H R V)$.

leaflets (including those of patients with critical pulmonary stenosis) varied between normal (Fig. 7a), moderate dysplasia (Fig. 7b), and severe dysplasia (Fig. 7c). There was little correlation between the degree of dysplasia and the size of the tricuspid annulus or of the right ventricular cavity, very dysplastic leaflets being found in some specimens with normal-sized right ventricular cavity and normal tricuspid annular circumference and nearly normal leaflets sometimes being associated with a tiny right ventricular cavity.

Ebstein's anomaly, defined as displacement of the effective atrioventricular orifice into the right ventricle, was present in 10 specimens. In 6 , the contiguous parts of the septal and inferior leaflets originated at a distance from the tricuspid annulus, the anterior leaflet arising normally (Fig. 8a). In 4 specimens, the tricuspid leaflets were replaced by a fibromuscular membrane extending from the anterior tricuspid annulus to the junction of the inlet with the outlet and trabecular portions of the right ventricle (Fig. 8b, c). In each of these the 'tricuspid' orifice consisted of a single small restrictive perforation in the membrane and was thus displaced far into the right ventricular cavity.

Tricuspid stenosis out of proportion to the right ventricular size was present in 7 cases. The obstruction was caused by heaped up dysplastic septal leaflet tissue in 2 cases of Ebstein's anomaly and appeared to be mild. In the cases of Ebstein's anomaly with a perforate membrane the 'tricuspid' obstruction was severe. In a sixth 


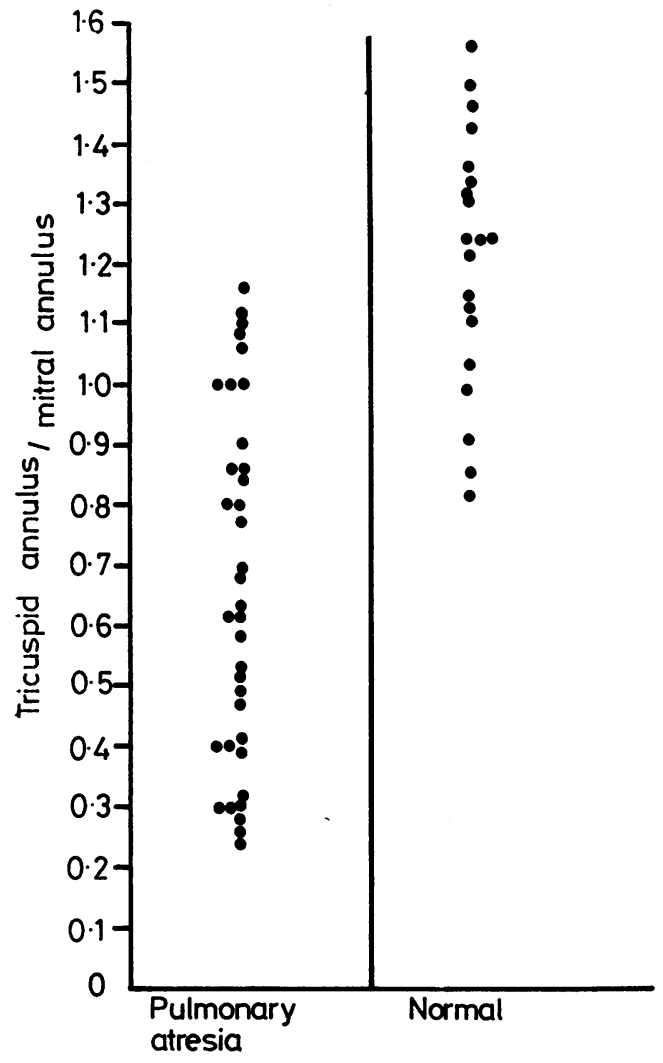

Fig. 6 Comparison of the relative tricuspid annular size in normals and in specimens with pulmonary atresia and intact ventricular septum.
Table Incidence of Ebstein's anomaly and of varying degrees of dysplasia of the tricuspid leaflets in pulmonary atresia.

\begin{tabular}{llllll}
\hline & \multicolumn{4}{l}{$R V$ cavity } \\
\cline { 2 - 5 } & Tiny & Small & Normal & Large & Total \\
\hline Ebstein's & 5 & 3 & & 2 & 10 \\
Tricuspid leaflets & & & 1 & & 4 \\
$\quad$ Very dysplastic & 3 & 5 & 2 & & 7 \\
Moderately thick & 2 & 4 & 5 & 1 & 11 \\
Slightly thick & 2 & 1 & 1 & 1 & 5 \\
\hline Normal & 2 & & & & \\
\hline
\end{tabular}

specimen with severe tricuspid stenosis, both anterior and septal leaflets attached directly to a single anterior papillary muscle, constituting a 'parachute' tricuspid valve. In the final case the tricuspid valve was 'unicuspid', the sole commissure being an inferior one.

In 4 cases the tricuspid valve gave every impression of having been regurgitant during life. The right ventricle was large in 3 of these and normal-sized in 1 . In 2 of the 4, regurgitation was related to Ebstein's anomaly. In the third case the tricuspid leaflets were somewhat thickened but were well formed; there was slight dilatation of the tricuspid annulus and severe dilatation of the right atrium. In the fourth specimen the tricuspid annulus was hugely dilated and the right ventricle large and very thin-walled.

In 11 of the specimens there was convex bulging of the left ventricular septal surface. Whether

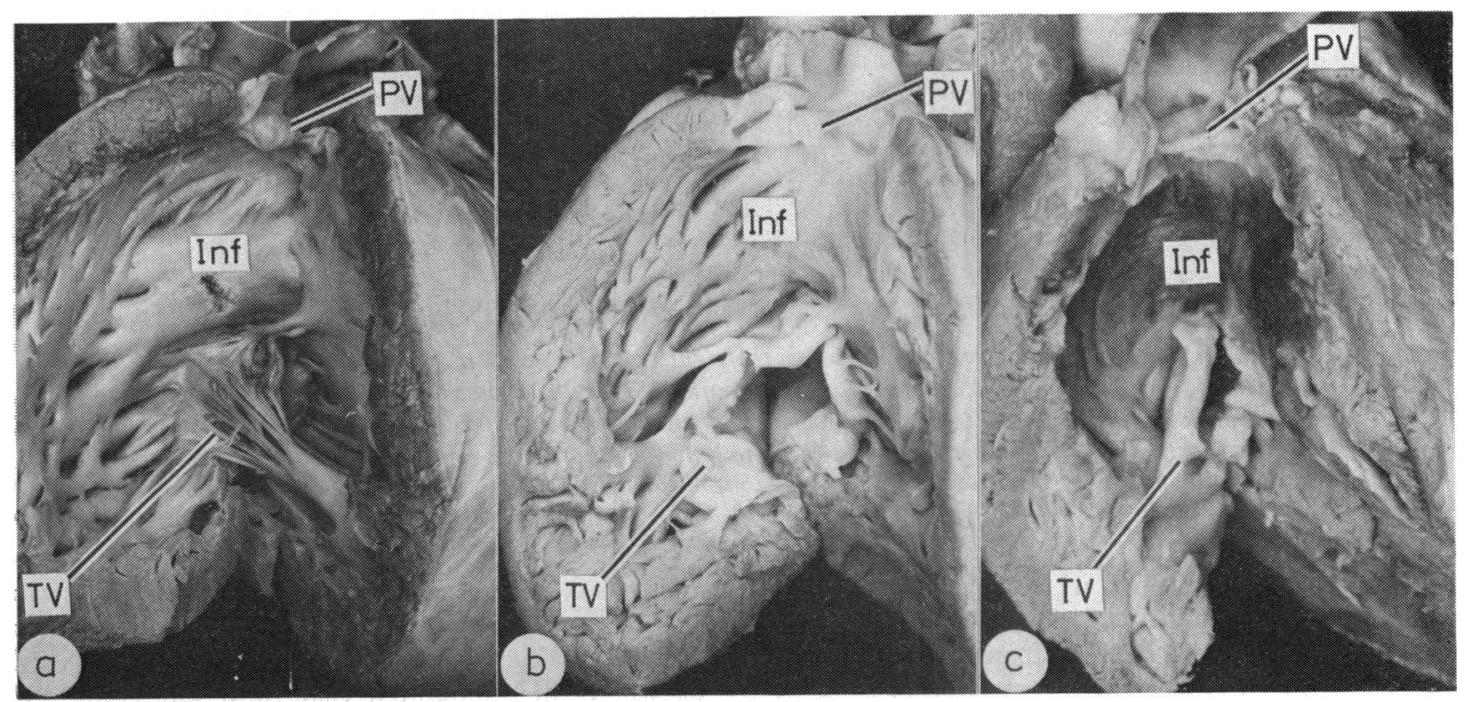

Fig. 7 Tricuspid leaflets in hearts with critical pulmonary stenosis and pulmonary atresia. (a) Normal (pulmonary stenosis). (b) Moderately dysplastic (pulmonary stenosis). (c) Very dysplastic (pulmonary atresia). 


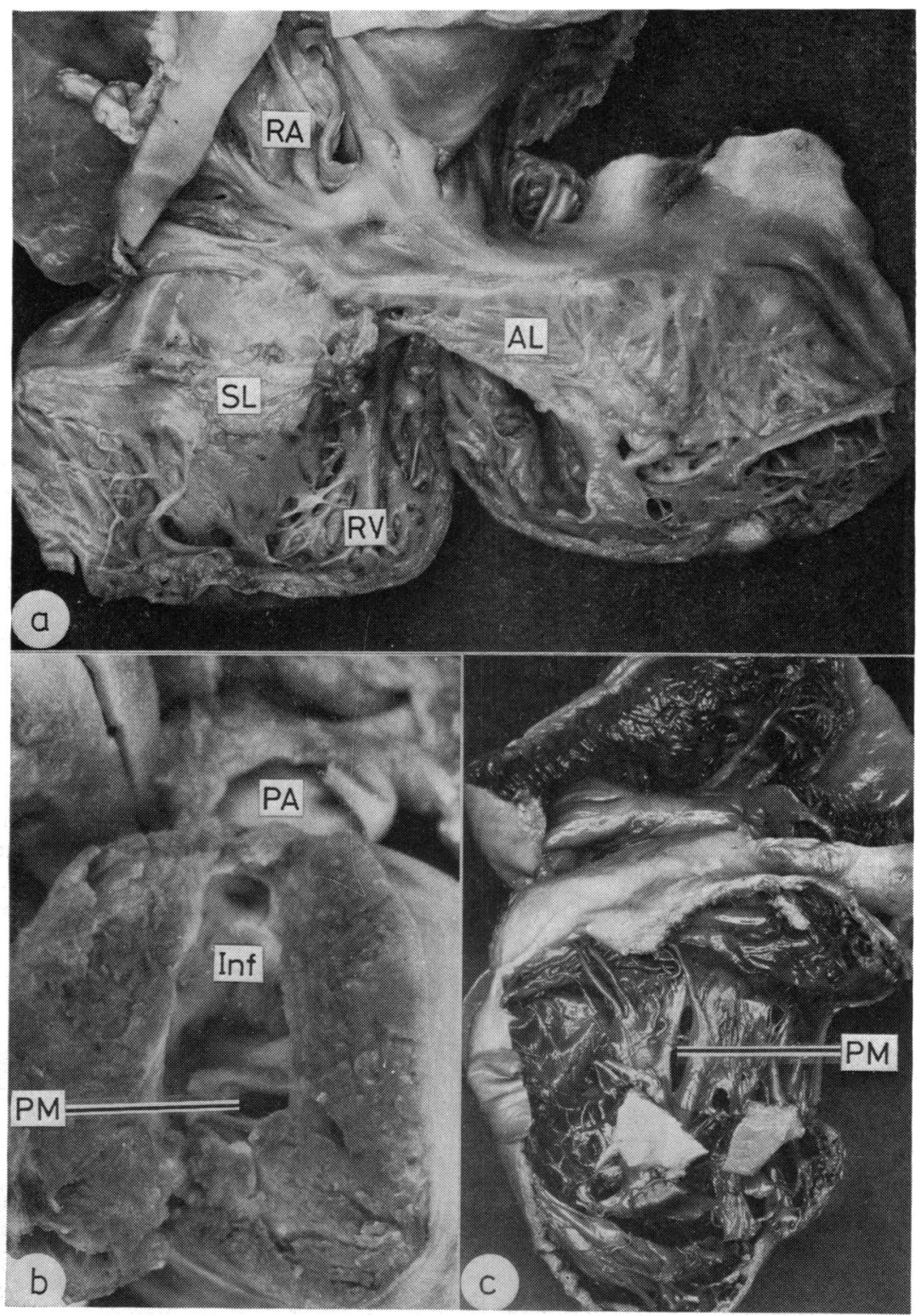

Fig. 8 Ebstein's anomaly. (a) $A$ view of the right ventricle showing a large anterior tricuspid leaflet $(A L)$ and a displaced septal leaflet $(S L)$. The right ventricle is moderately dilated and thin-walled. (b) A perforate membrane (PM) viewed from the right ventricular outlet portion (Inf.). (c) The anterior wall of the right ventricle has been removed to display a muscularised perforate membrane (PM).

this could have resulted in subaortic obstruction in any case is conjectural. No discrete subaortic stenosis was seen. Both left ventricular fibroelastosis and an apical aneurysm were present in a single case. The left anterior descending coronary artery was large in 11 specimens, and the posterior descending in 2. The mitral and aortic valves were normal in all specimens. The ductus arteriosus was patent in all unoperated cases and was commonly long and tortuous. 


\section{Discussion}

Previous observers have disagreed concerning the precise nature of right ventricular hypoplasia in pulmonary atresia with intact septum. Some have argued that cavity size can simply be divided into 'small' and 'large' (Greenwold et al., 1956). Others contend that a spectrum of cavity size is seen (Gersony et al., 1967; Bowman et al., 1971; Murphy et al., 1971). We have tried to obtain objective estimation of this feature by measuring ventricular cavity size and comparing it with similar measurements in the normal heart, as more recently have Freedom et al. (1978). Since the size of each measured right heart structure relative to that particular heart seemed more likely to be meaningful than the absolute dimensions themselves, each right heart measurement was related to its left heart counterpart. Each heart, in effect, therefore served as its own control. Such comparisons also lessened the possible artefactual effect of fixation since right and left heart structures should have been equally affected. The results showed that while the specimens were subjectively divided into groups with tiny, small, normal, and dilated right ventricular cavities, the measurements indicated a spectrum of cavity size which overlapped the normal values. These results are in accord with those of Freedom et al. (1978).

The size of the right ventricular cavity is the most obvious limiting factor in immediate survival after valvotomy. In the 12 cases with a tiny right ventricle the cavity size alone would have precluded survival after valvotomy done as the sole surgical procedure, the accompanying infundibular atresia or stenosis compounding the situation. Though there have been isolated reports of right ventricular growth after valvotomy (Moller et al., 1970; Bowman et al., 1971; Murphy et al., 1971; Luckstead et al., 1972), there is currently no way of predicting growth potential. The extreme hypoplasia of these tiny right ventricles, the difficulty of outflow tract reconstruction in a newborn infant, and the probable extremely limited potential for growth and function are strong arguments against such a procedure in this group.

Although valvotomy might have been feasible in patients in the small right ventricle group (save the one specimen with infundibular atresia), the degree of infundibular stenosis common to these patients would have necessitated infundibular resection and/or patching, either as a primary procedure (Rigby et al., 1977) or at subsequent surgery (Bowman et al., 1971). In this group the potential for right ventricular growth may be better, but unless the usually associated small tricuspid annulus were to grow as well, tricuspid stenosis would remain (Trusler et al., 1976), and would probably presage a poor prognosis. The cases with dilated right ventricles were also poor candidates for reconstruction; therefore only the cases with near to normal ventricular size could be considered as having a good morphological prognosis.

In the group as a whole the tricuspid valve was the second most common factor limiting the feasibility of success of pulmonary valvotomy. In 5 cases there was a degree of tricuspid stenosis which would have severely limited ventricular inflow, even if a good outflow could have been established. In at least 2 other cases there seemed to have been such severe tricuspid regurgitation that even complete relief of the right ventricular outlet obstruction might not have resulted in a right ventricular outflow sufficient to sustain life. It did not seem likely that either severe tricuspid stenosis or tricuspid regurgitation could have been effectively treated surgically, since relief of inlet obstruction in cases with severe tricuspid stenosis seemed impossible short of resecting the tricuspid valve (or the perforate membrane in the cases with Ebstein's anomaly). A further compounding factor must surely have been the state of the ventricular myocardium. Though an adequate right ventricular cavity and infundibulum, a functional tricuspid valve, and a sizeable pulmonary artery satisfy the plumbing requirements of a successful valvotomy, an effective pump is also required. The right ventricular endocardial fibroelastosis found in 25 per cent of the specimens in our series may have deleteriously influenced myocardial function.

In contrast, the size of the main pulmonary artery was not a limiting factor, only 3 cases being judged too small to have accepted an effective right ventricular output. In all 3, the right ventricular cavity was additionally tiny.

In summary, our anatomical study has shown a range of morphological features in pulmonary atresia with intact septum which shows that surgical treatment must be tailored to each individual case. Thus, pulmonary valvotomy did not seem a feasible procedure in half of the cases in this series. Such cases may benefit from an aortopulmonary anastomosis since in the majority of hearts the pulmonary arteries were found to be of good size. Subsequent repair may be possible by constructing a right atrial to pulmonary artery conduit. Only the cases with a near to normal sized right ventricular cavity seemed anatomically suited to complete repair. Similar cases should probably be treated by pulmonary valvotomy as an initial procedure. Our measurements show that 
the size of the tricuspid annulus bears a positive correlation with cavity size, and this may be a factor of clinical value.

We are grateful to our clinical colleagues who permitted us to study patients previously in their care, namely, Drs. M. C. Joseph, E. A. Shinebourne, J. L. Wilkinson, and O. Scott. Drs. I. M. Gerlis and J. A. Partridge drew our attention to some of the cases studied, and Dr. A. E. Becker made it possible for us to examine the normal hearts. The investigation was supported by a grant from the British Heart Foundation.

\section{References}

Bowman, F., Malm, J., Hayes, C., Gersony, W., and Ellis, K. (1971). Pulmonary atresia with intact ventricular septum. Fournal of Thoracic and Cardiovascular Surgery, 61, 85-95.

Celermajer, J., Bowdler, J. D., Gengos, D., Cohen, D., and Stuckey, D. (1968). Pulmonary valve fusion with intact ventricular septum. American Heart fournal, 76, 452-465.

Davignon, A. L., Greenwold, W. E., DuShane, J. W., and Edwards, J. E. (1961). Congenital pulmonary atresia with intact ventricular septum: clinicopathologic correlation of two anatomic types. American Heart fournal, 62, 591-602.

Freedom, R. M., Dische, M. R., and Rowe, R. D. (1978). The tricuspid valve in pulmonary atresia and intact ventricular septum. Archives of Pathology and Laboratory Medicine, 102, 28-31.

Gersony, W., Bernhard, W., Nadas, A., and Gross, R. (1967). Diagnosis and surgical treatment of infants with critical pulmonary outflow obstruction. Circulation, 35, 765-776.

Greenwold, W. E., DuShane, J. W., Burchell, H. B., Bruwer, A., and Edwards, J. E. (1956). Congenital pulmonary atresia with intact ventricular septum: two anatomic types (abstract). Circulation, 14, 945-946.

Luckstead, E., Mattioli, L., Crosby, I., Reed, W., and Diehl, A. (1972). Two stage palliative surgical approach for pulmonary atresia with intact ventricular septum (Type 1). American fournal of Cardiology, 29, 490-496.

Moller, J. H., Girod, D., Amplatz, K., and Varco, R. L. (1970). Pulmonary valvotomy in pulmonary atresia with hypoplastic right ventricle. Surgery, 68, 630-634.

Murphy, D. A., Murphy, D. R., Gibbons, J. E., and Dobell, A. R. C. (1971). Surgical treatment of pulmonary atresia with intact ventricular septum. Fournal of Thoracic and Cardiovascular Surgery, 62, 213-219.

Rigby, M. L., Silove, E. D., Astley, R., and Abrams, L. D. (1977). Pulmonary atresia with intact ventricular septum: open heart surgical correction at 32 hours. British Heart fournal, 39, 573-576.

Trusler, G. A., Yamamoto, N., Williams, W. G. Izukawa, T., Rowe, R., and Mustard, W. T. (1976). Surgical treatment of pulmonary atresia with intact ventricular septum. British Heart Fournal, 38, 957-960.

Requests for reprints to $\mathrm{Dr}$ R. H. Anderson, Department of Paediatrics, Brompton Hospital, Fulham Road, London SW3 6HP. 